\title{
Anonymizing Location Information in Unstructured Text Using Knowledge Graph
}

\author{
Taisho Sasada \\ Nara Institute of Science and \\ Technology \\ 8916-5 Takayama, Ikoma, \\ Nara 6300 192, Japan \\ sasada.taisho.su0@is.naist.jp
}

\author{
Yuzo Taenaka \\ Nara Institute of Science and \\ Technology \\ 8916-5 Takayama, Ikoma, \\ Nara 6300 192, Japan \\ yuzo@is.naist.jp
}

\author{
Youki Kadobayashi \\ Nara Institute of Science and \\ Technology \\ 8916-5 Takayama, Ikoma, \\ Nara 6300 192, Japan \\ youki-k@is.naist.jp
}

\begin{abstract}
There is a growing need to anonymize data as new businesses are increasingly utilizing vast amount of unstructured text. Also, unstructured text have a risk of personal location estimation by considering location information. Nevertheless, existing generalizations do not take into location information and therefore cannot robustly handle this attack. In this study, we proposed anonymizing location information in unstructured text using knowledge graph newly constructed from an actual geographic information system. Our method has the advantages of anonymization, taking into account actual geographic information, handling abbreviations and spelling inconsistencies, and allowing for dynamic graph updates. The results of the evaluation experiments show that anonymization is more robust than existing methods against location estimation attacks without compromising its usefulness as a dataset. Also, we found that the names of organizations and places with a high probability of occurrence in unstructured text are more likely to lead to personal identification.
\end{abstract}

\section{CCS Concepts}

- CCS $\rightarrow$ Security and privacy $\rightarrow$ Security services $\rightarrow$ Pseudonymity, anonymity and untraceability

\section{Author Keywords \\ Unstructured Text, Privacy Preserving, Generalization, Knowledge Graph}

\section{INTRODUCTION}

As the spread of Consumer Generated Media (CGM) such as blogs, web pages, and message bords, unstructured text have been one of important information for understanding peoples' attributes. These data is being provided to thirdparty organizations providing targeting businesses. When providing data to a third party, it is necessary to remove not only personal information such as name, birth dates, etc. but Permission to make digital or hard copies of all or part of this work for personal or classroom use is granted without fee provided that copies are not made or distributed for profit or commercial advantage and that copies bear this notice and the full citation on the first page. Copyrights for components of this work owned by others than ACM must be honored. Abstracting with credit is permitted. To copy otherwise, or republish, to post on servers or to redistribute to lists, requires prior specific permission and/or a fee. Request permissions from permissions@ acm.org.

iiWAS'20, The 22nd International Conference on Information Integration and Webbased Applications \& Services, November 30-December 2, 2020, Chiang Mai, Thailand

() 2020 ACM. ISBN $978-1-4503-8922-8 / 20 / 11 \ldots \$ 15.00$

DOI: https://doi .org/10.1145/3428757.3429195 also other sensitive information, which implies individuals, so as to prevent identifying individuals. Several data may sometimes fill lacked information by each other and together allow to identify individuals. Such kind of data is called "quasiidentifiers". Thus, quasi-identifiers cause serious problems in data trading market.

There are two methods of anonymizing quasi-identifiers: suppression and generalization [6]. Suppression is to replace quasi-identifiers with symbols such as $*$ and $\times$. Although suppression is considerably effective to completely remove quasi-identifiers from data, it inevitably drops the essential value of data due to the nature of that process. On the other hand, the other type of method, generalization, replace a quasiidentifier with abstracted information but keeps intrinsic value of data; this prevents data being useless. These generalizations allow us to provide unstructured text with a third party, but these texts are vulnerable to Location Estimation Attacks to identify a personal location.

In the existing research [9], the generalization of geographical quasi-identifiers has been studied using WordNet, an English conceptual dictionary, which searches for synonyms and words corresponding to higher-level concepts. They generalize the expression by WordNet to deal with abbreviations and spelling inconsistencies of geographical quasi-identifiers in unstructured text, while the generalization protects the privacy of individuals. But, WordNet is not built by considering actual geographic information. Therefore, WordNet cannot adequately handle Location Estimation Attacks for personal identification. Moreover, the superordinate and subordinate relationships of the words in WordNet are geographically ambiguous. Therefore, generalization by WordNet can cause serious information loss in the unstructured text. This may lead to a bloated impact on the results of the analysis. The actual addresses are arranged in a hierarchical order: block number, street name, city, state, and country. Nevertheless, WordNet does not consider this hierarchical relationship, therefore WordNet may directly generalize city name to country names. To prevent serious information loss, it is necessary to anonymize data by generalization using precise conceptual relationships in consideration of actual geographical information.

In this study, we propose anonymization of unstructured text by a precise generalization of attribute information contained in the names of organizations and places from a geographic 
information system and knowledge graph based on the information. The contributions made by this study are as follows:

- Elaborate generalizations that take into account actual geographic information

- Dealing with abbreviations and spelling inconsistencies of identifiers

- Dynamically update the knowledge graph

The structure of this paper is as follows. First, we give an overview of related research on generalization and its problems in Section 2. In Section 3, we describe the attack model targeted in this study to clarify the privacy that can be protected. In Section 4, we describe the content of the proposals made to address the existing problems. In Section 5, we describe the contents of evaluation experiments performed to verify the robustness and usefulness of the proposed method and a discussion of the results. In Section 6, we describe the summary of this study and future issues.

\section{RELATED WORKS}

In this section, we describe a way of generalizing quasiidentifiers by using Named Entity Recognition and WordNet.

Named Entity Recognition is a natural language processing task, but it is also used in privacy research [2]. Named Entity are expressions that describe the name of a specific person, thing, place, etc. Individuals can be re-identified from the Named Entities in a text even after the identifier is removed. To date, a study have been carried out to suppress or generalize the extracted Named Entity for anonymous processing [12].

Hassan et al. constructed a quasi-identifier dictionary based on all extracted named entities [3]. They generalize words in the quasi-identifier dictionary by replacing with tags. As named entities include both quasi/non-quasi identifiers, intrinsic information of non-quasi-identifiers will be lost. Hence, in order to generalize quasi-identifier by Named Entity Recognition, it is necessary to select words that actually comprise of quasi-identifiers. Also, in cace of generalizing quasiidentifiers, the synonymy and similarity of words should be taken into account to prevent re-identification and to avoid significant changes in the results of analysis after generalizing quasi-identifiers [10].

Nguyen-Son et al. generalize discriminative words by replacing them with synonyms and superordinate words stored in WordNet [7] and showed the usefulness of using conceptual relations in anonymization [9]. However, WordNet is not a dictionary built with consideration for actual positional relationships in geographic words. Therefore, generalization by WordNet cannot robustly deal with location estimation attack. Besides, it is difficult to replace unknown words, to update it dynamically, and replace it with new geographical words or organization words. Thus it is necessary to construct a conceptual dictionary based on the positional relationships of the extracted expressions when generalizing using the conceptual dictionary.

\section{ATTACK MODEL}

In this section, we describe the case where an attacker performs table linkage using a location estimation classifier.

\subsection{Personal identification}

One of attack models is record linkage [10]. Record linkage is uniquely identifying individuals by combining identifiers (quasi-identifiers) in data and leaks the private information. Such personal identification can be handled by anonymizing the data to satisfy $k$-anonymity.

But, in the case of unstructured text, it is difficult to preprocess the text to satisfy $k$-anonymity because it is not known where the quasi-identifier is located in the text. Besides, if an attacker has prior background knowledge that is already public, it is possible that a unique identifier can be created by merging those data. This attack model is called table linkage. Figure 1 shows an example where each user posts their review of a restaurant. An attacker combines public information and prior background knowledge to infer a quasi-identifier, which is deleted private information, and thus an individual is identified.

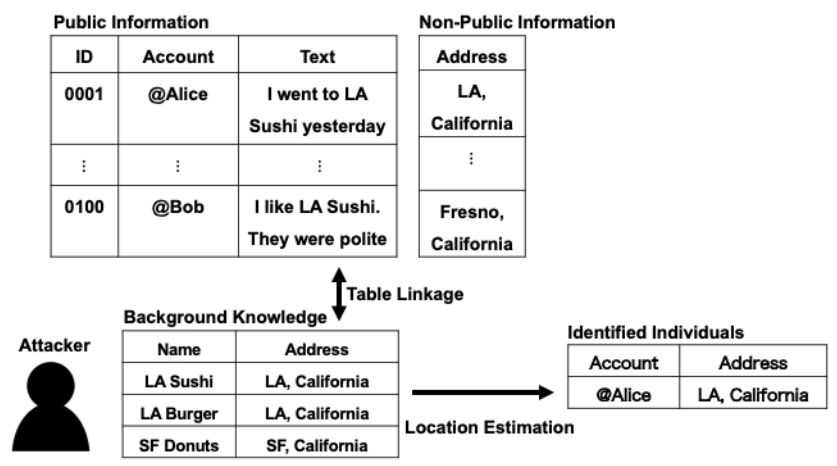

Figure 1. Quasi-identifier presumption attack based on the attacker's prior background knowledge

\subsection{Location estimation attack in unstructured text}

As explained in Section 3.1, unstructured text can identify individuals based on prior background knowledge and data that are already publicly available. Hence, we present an account of location estimation attack to validate the data with identifiers and quasi-identifiers removed from the data in terms of robustness and usefulness.

Mahmud et al. create a word document matrix from sentences published on Twitter and estimate the location of individuals by probabilistic attacks with an ensemble model trained with that matrix and the assigned Geo tags [5]. Estimations were made at the city and state level, and the correct rates were $58 \%$ and $66 \%$, respectively.

\section{PROPOSED METHOD}

In this section, we explain the location estimation classifier, the construction of the knowledge graph, and the generalization method used in the proposed method.

\subsection{Extraction of quasi-identifiers}

In this study, the names of places and organizations are assumed to be quasi-identifiers. For Named Entity Recognition, 
we use TwitterNER which is capable of extracting the name of place and organization from tweets with the highest accuracy [8]. However, as explained in Section 3.1, not all names of places and organizations are quasi-identifiers. In unstructured text, the names of places and organizations are sometimes used in contexts unrelated to the user, such as information about a travel destination in social media or a sales source in review texts. In such cases, anonymization rather makes data useless.

Therefore, we first select unstructured text that may be possibly used for location estimation. From the selected unstructured text, we extract further Named Entities, and identify only the names of places and organizations that need to be anonymized by generalization as quasi-identifiers. This generalizes only the names of places and organizations in the selected unstructured text, which minimizes the generalization targets and prevents a decrease in the usefulness of the data. For estimation, we convert each user's unstructured text into a word-document matrix and train from it to construct model. The constructed model estimates the location of each unstructured text and extracts unstructured text that needs to be anonymized if the estimation results are correct.

\subsection{Construction of a quasi-identifier dictionary}

First, let $d_{i}^{q i d}$ be the $i$ th unstructured text that needs to be anonymized because it contains identifiable quasi-identifiers, and $w_{j}^{\text {qid }}$ be the $j$ th quasi-identifier included in this $d_{i}^{q i d}$ unstructured text. If we construct a dictionary with the unstructured text selected in Section 4.1 as a key, and the names of places and organizations as the value, the same names of places and organizations may repeatedly appear in different unstructured texts. And it is inefficient in terms of the number of times to perform the same generalization process each time. Therefore, in this study, we realize quasi-identifier retrieval in an unstructured text set containing the same quasi-identifiers, and improve the efficiency of anonymity processing by generalization.

Then, in order to eliminate the duplication of values, we try to search for quasi-identifiers by inverted indexes based on the created indexes. An inverted index allows the mapping of words that appear in a document to documents. The construction of a quasi-identifiers dictionary $D^{\text {qid }}$, which links quasi-identifiers and unstructured text with an inverted index, eliminates the duplication of quasi-identifiers, and by referring to Value, the same processing can be performed on quasi-identifiers existing in different unstructured texts, thus making anonymity processing more efficient. Table 4.2 shows an example of quasi-identifiers dictionary.

\subsection{Generalization using Knowledge Graph}

A knowledge graph represent knowledge about an event as connected graph, which can represent geographical representations and categories of organizational names obtained from Google Maps [1]. Figure 2 shows an example of a knowledge graph for the category name and address of University of California, Los Angeles. This knowledge graph provides a hierarchical representation of UCLA's addresses, allowing us to precisely define the superordinate and subordinate relationships between the place names contained in the addresses.
Table 1. quasi-identifier dictionary $D^{\text {qid }}$

\begin{tabular}{|c|c|}
\hline Index $\left[w_{i j}^{q i d}, j\right]$ & Item $\left[\mathrm{id}_{i}: d_{i}^{q i d}\right]$ \\
\hline [LA Park, 1] & $\begin{array}{l}\text { [1: LA Park is known for } \\
\text { its mind-blowing rides] }\end{array}$ \\
\hline [Griffith Park, 1] & $\begin{array}{l}{[2: \text { Griffith Park, in the eastern }} \\
\text { part of the Santa Monica Mountains }]\end{array}$ \\
\hline $\begin{array}{l}{[\text { Sant: }} \\
\text { Moun }\end{array}$ & $\begin{array}{l}\text { [2: Griffith Park, in the eastern } \\
\text { part of the Santa Monica Mountains] }\end{array}$ \\
\hline$[\operatorname{Los} A$ & $\begin{array}{l}\text { [3: Just outside Los Angeles, } \\
\text { Disneyland is California's premier } \\
\text { family vacation destination] }\end{array}$ \\
\hline [Disneyland, 2] & $\begin{array}{l}\text { [3: Just outside Los Angeles, } \\
\text { Disneyland is California's premier } \\
\text { family vacation destination] }\end{array}$ \\
\hline [Cali & $\begin{array}{l}\text { [3: Just outside Los Angeles, } \\
\text { Disneyland is California's premier } \\
\text { family vacation destination] }\end{array}$ \\
\hline
\end{tabular}

In this study, we construct a knowledge graph in the form of a Property Graph Model [11] in which each node $V$ is composed of a set of edges $\mathcal{E}$, since each node has multiple properties. The procedure for constructing a knowledge graph is shown in the algorithm 1 . This knowledge graph is a labeled and attributed graph $\mathcal{G}$ consisting of a set of nodes $\mathcal{V}$ and a set of edges $\mathcal{E}$, denoted by $\mathcal{G}=(\mathcal{V}, \mathcal{E}, \mathcal{L} \mathcal{V}, \mathcal{L} \mathcal{E})$. The graphs are stored in the native graph database, $\mathrm{Neo} 4 \mathrm{j}$.

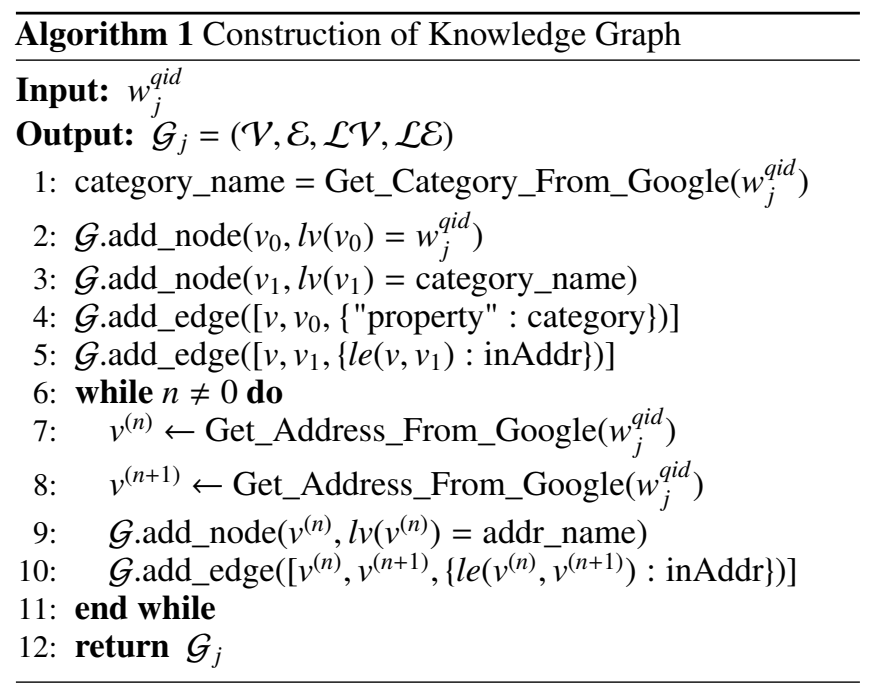

\subsection{Generalization with Knowledge Graph}

To deal with the location estimation attack described in Section 3 , it is necessary to generalize the quasi-identifier dictionary $D^{\text {qid }}$ to a representation where value is not estimable. We generalize quasi-identifiers to a superordinate representation using the knowledge graph constructed in Section 4.3 for the value of the dictionary of quasi-identifiers explained in Section 4.2. The generalization process by knowledge graph is shown in Algorithm 2. In lines 1-4, we issue a set of category names stored in $V_{1}$ and an address stored in $v^{(n)}$ as a query $Q$ on Google Maps. If the number of query results is 2 or more, we store $Q$ to $w_{j}^{k}$ in line 5 . This process is performed on each 


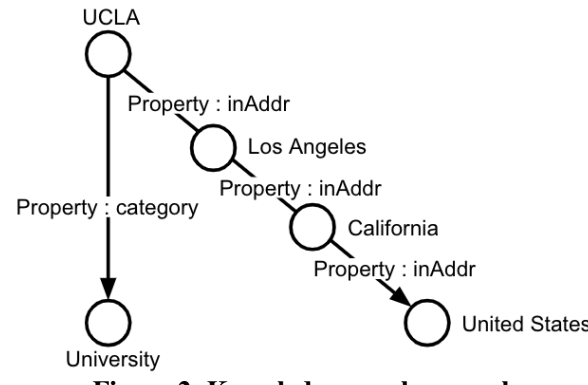

Figure 2. Knowledge graph example

value of the quasi-identifier dictionary $D^{\text {qid }}$ to anonymize all unstructured text. This set of generalizations allows for precise anonymization that preserves its usefulness as a dataset while improving its robustness to location estimation attack.

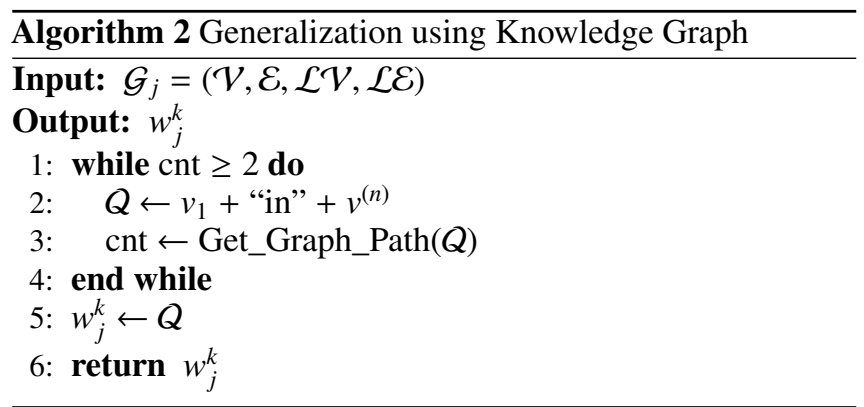

\section{EXPERIMENTS}

We describe the experiments to evaluate the robustness against location estimation attack and the usefulness as a dataset.

\subsection{Dataset, Model, and Evaluation metric}

To validate the robustness to location estimation and the usefulness as a dataset for data analysis, we evaluate it using Gender Classifier Data ${ }^{1}$. This dataset contains information on each user's attributes such as username, location, gender, and so on, as well as Twitter. Using this dataset, we perform location estimation attack and gender classification based on machine learning with tweet data, and examine the effects on the F1-Score before and after anonymization. The smaller the F1-Score in robustness, the more robust dataset is to location estimation attack. And if the F1-Score in usefulness is not different from the raw dataset, it means that the usefulness is not degraded. Also, we use four types of models with default parameters: Random Forest, Decision Tree, Logistic Regression, Support Vector Machine, and Neural Network. Moreover, as an evaluation metric, we use the F1-Score obtained by the location estimation attack before and after the generalization by the existing and proposed methods. Experiments were conducted in Cross-Validation ( $\mathrm{CV}$ ) to evaluate the performance of the constructed classifier. Since the number to be divided is generally set to 10 [4], we perform 10 -fold CV in this study.

\subsection{Experimental Results}

Table 2 left side (Robustness) shows the F1-Score of the location estimation on three different datasets: raw dataset (RD),

\footnotetext{
${ }^{1}$ https://data.world/crowdflower/gender-classifier-data
}

generalized dataset with WordNet (WN) as existing method, and generalized dataset with Knowledge Graph (KG) as proposed method. This result was obtained by the most accurate Random Forest. The results of the location estimation attack showed that the F1-Score of the location estimation attack in the proposed method was smaller than that of the existing methods in all subsets.

In the case of WordNet, quasi-identifiers are replaced by abbreviations. But as an example, if attacker query Google Maps with "University of CA, LA", attacker can identify that "University of CA, LA" is "University in Los Angeles" before generalization. Therefore, we see that this representation is insufficient as a generalization to prevent location estimation. On the other hand, the proposed method generated by the "University in Los Angeles" will return 256 as a candidate in the search results, so the query with the notation The probability of being identified when taking into account location information is $1 / 256(\fallingdotseq 0.0039)$. In short, WordNet cannot generalize to take into account actual location information, thus some of the generalized representations could be uniquely identified if considering location information. On the other hand, the proposed method can generalize for preventing unique identification by considering the location information. Hence, F1-Score is lower on the generalization by Knowledge Graph (proposed method) against location estimation attack compared to the generalization by WordNet (existing methods). The small F1-Score means that a location estimation attack on the generalized dataset cannot identify the correct location of the tweet, which makes it difficult to estimate the user's tweet location even if the dataset is provided to a third party.

Also, Table 2 right side (Usefulness) shows the F1-Score of the gender classification. $S_{1}$ to $S_{10}$ are 10 subsamples created by the 10 -fold $\mathrm{CV}$. The results of gender classification did not change significantly before and after generalization. Changelessness means that the generalization of quasi-identifiers by the proposed method does not diminish the usefulness as the dataset. In short, a third party can use dataset for analysis and training with machine learning models after generalization.

Figure 3 shows the probability of occurrence of each quasiidentifier in an unstructured text and the value distribution of $k$ when the quasi-identifier is generalized by proposed method. The higher the frequency of place names organization names, the greater the value of $k$ at generalization. Thus, since quasiidentifiers with a high probability of occurrence are more likely to lead to the identification of individuals, it is necessary to generalize to a more abstract expression than to quasi-identifiers with a low probability of occurrence.

\subsection{Discussion}

In addition to anonymization to counter location estimation attack, the proposed method also generalizes the names of places and organizations in the unstructured text before posting to prevent identification from being posted on social media and CGN. Also, our method can be applied to Geographic Information Retrieval because we have defined upper-order relations and lower-order relations of addresses in consideration of actual geographic information. 
Table 2. Results of Classifying User Gender Using 10-fold CV

\begin{tabular}{l|cccc|ccc}
\hline & \multicolumn{4}{|c}{ Robustness } & \multicolumn{3}{c}{ Usefulness } \\
\hline \multirow{4}{*}{ S1 } & & RD & WN & KG & RD & WN & KG \\
& City & 0.58 & 0.48 & $\mathbf{0 . 2 1}$ & $\mathbf{0 . 5 6}$ & 0.48 & 0.55 \\
& State & 0.63 & 0.54 & $\mathbf{0 . 4 3}$ & & & \\
S2 & City & 0.54 & 0.43 & $\mathbf{0 . 2 8}$ & $\mathbf{0 . 5 7}$ & 0.49 & 0.52 \\
& State & 0.61 & 0.49 & $\mathbf{0 . 4 2}$ & & & \\
S3 & City & 0.52 & 0.47 & $\mathbf{0 . 2 4}$ & 0.47 & $\mathbf{0 . 5 1}$ & 0.48 \\
& State & 0.66 & 0.53 & $\mathbf{0 . 4 5}$ & & & \\
S4 & City & 0.55 & 0.46 & $\mathbf{0 . 2 0}$ & $\mathbf{0 . 5 9}$ & 0.51 & 0.54 \\
& State & 0.60 & 0.58 & $\mathbf{0 . 4 1}$ & & & \\
S5 & City & 0.51 & 0.42 & $\mathbf{0 . 2 1}$ & $\mathbf{0 . 5 1}$ & 0.43 & 0.50 \\
& State & 0.63 & 0.59 & $\mathbf{0 . 4 5}$ & & & \\
S6 & City & 0.54 & 0.48 & $\mathbf{0 . 2 3}$ & $\mathbf{0 . 5 4}$ & 0.48 & 0.53 \\
& State & 0.62 & 0.6 & $\mathbf{0 . 4 4}$ & & & \\
S7 & City & 0.56 & 0.41 & $\mathbf{0 . 1 9}$ & $\mathbf{0 . 5 6}$ & 0.41 & 0.52 \\
& State & 0.63 & 0.49 & $\mathbf{0 . 4 8}$ & & & \\
S8 & City & 0.53 & 0.45 & $\mathbf{0 . 2 7}$ & $\mathbf{0 . 5 3}$ & 0.45 & 0.48 \\
& State & 0.60 & 0.61 & $\mathbf{0 . 4 5}$ & & & \\
S9 & City & 0.51 & 0.46 & $\mathbf{0 . 1 8}$ & $\mathbf{0 . 5 1}$ & 0.46 & $\mathbf{0 . 5 1}$ \\
& State & 0.59 & 0.57 & $\mathbf{0 . 4 9}$ & & & \\
S10 & City & 0.54 & 0.42 & $\mathbf{0 . 2 3}$ & $\mathbf{0 . 5 4}$ & 0.42 & 0.52 \\
& State & 0.61 & 0.53 & $\mathbf{0 . 4 5}$ & & & \\
\hline
\end{tabular}

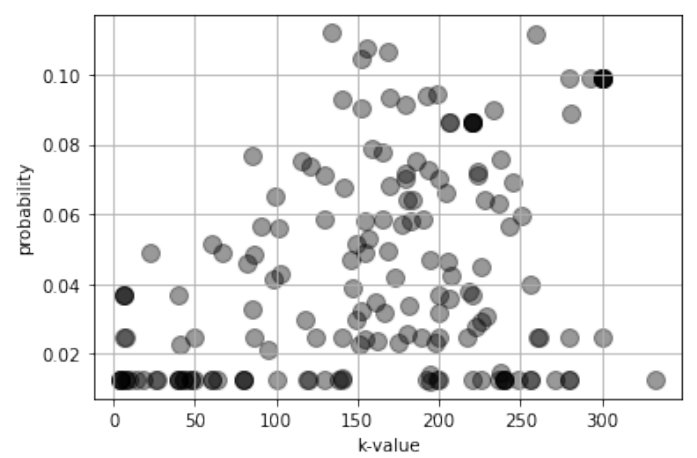

Figure 3. Frequency of quasi-identifiers and value distribution of $k$

On the other hand, generalizing all the names of places and organizations in identifiable unstructured text may produce unnatural text. Unnatural sentences may convey different meanings to the users, so it is necessary to verify the naturalness of the sentences. Also, we found that it is not possible to deal with location estimation attack based on product names or specific product prices, even if they are not the names of places or organizations. Location estimation attack are not necessarily only textual. For example, there are cases in which external information such as images and time. Therefore, it is necessary to generalize to quasi-identifiers other than place names and organizational names, such as product names, product prices, images, and time.

\section{CONCLUSION}

In this paper, we proposed an anonymization method by constructing knowledge graph from actual geographic information systems to protect the privacy of unstructured text. This is because there is a risk of location estimation attack but also a growing need to anonymize unstructured text for sharing it with a third party. We generalized the names of places and organizations using the constructed knowledge graph. Unlike the existing methods, we generalized quasi-identifiers while considering actual geographic information. As a result, experimental evaluation showed that our generalization is more robust to location estimation attack than existing methods while without diminishing its usefulness as a dataset.

In future work, we should address two issues. The first is to experiment with unstructured text other than tweets. We need to apply similar anonymization to review and other free text to verify whether they are robust against location estimation attack. The second is to consider how to process the names of organizations and geographies, which will lead to identification no matter how much they are generalized.

\section{Acknowledgement}

We thank Shuyo Nakatani from Cybozu Labs, Inc. for advice on writing this paper.

This work was supported in part by the ICS-CoE Core Human Resources Development Program, and in part by JSPS KAKENHI Grant Number JP18H03234.

\section{REFERENCES}

[1] Ballatore, Andrea and Wilson, David and Bertolotto, Michela. A survey of volunteered open geo-knowledge bases in the semantic web. In Quality issues in the management of web information, pages 93-120. Springer, 2013.

[2] A. Gkoulalas, G. Loukides, and J. Sun. Publishing data from electronic health records while preserving privacy: A survey of algorithms. Journal of biomedical informatics, 50:4-19, 2014.

[3] F. Hassan, J. Domingo-Ferrer, and J. Soria-Comas. Anonymization of Unstructured Data via Named-Entity Recognition. In International conference on modeling decisions for artificial intelligence, pages 296-305. Springer, 2018

[4] R. Kohavi et al. A Study of Cross-Validation and Bootstrap for Accuracy Estimation and Model Selection. In Ijcai, volume 14, pages 1137-1145. Montreal, Canada, 1995.

[5] J. Mahmud, J. Nichols, and C. Drews. Where Is This Tweet From? Inferring Home Locations of Twitter Users. International Conference on Weblogs and Social Media, 12:511-514, 2012.

[6] B. Mehta, U. P. Rao, R. Gupta, and M. Conti. Towards privacy preserving unstructured big data publishing. Journal of Intelligent $\mathcal{E}$ Fuzzy Systems, 36(4):3471-3482, 2019.

[7] G. A. Miller. WordNet: An Electronic Lexical Database. MIT press, 1998.

[8] S. Mishra and J. Diesner. Semi-supervised Named Entity Recognition in noisy-text. In Proceedings of the 2nd Workshop on Noisy User-generated Text, pages 203-212, 2016.

[9] H.-Q. Nguyen-Son, M.-T. Tran, H. Yoshiura, N. Sonehara, and I. Echizen. Anonymizing Personal Text Messages Posted in Online Social Networks and Detecting Disclosures of Personal Information. IEICE TRANSACTIONS on Information and Systems, 98(1):78-88, 2015.

[10] H. Pang, J. Shen, and R. Krishnan. Privacy-preserving similarity-based text retrieval. ACM Transactions on Internet Technology, 10(1):1-39, 2010 .

[11] I. Robinson, J. Webber, and E. Eifrem. Graph Databases. " O'Reilly Media, Inc.", 2013.

[12] D. Terzi, R. Terzi, and S. Sagiroglu. A Survey on Security and Privacy Issues in Big Data. In 2015 10th International Conference for Internet Technology and Secured Transactions, pages 202-207. IEEE, 2015. 